\title{
Construction of Small World Networks Based on K-Means Clustering Analysis
}

\author{
Jianyu $\mathrm{Li}^{1}$, Rui Lv ${ }^{1}$, Zhanxin Yang ${ }^{1}$, Shuzhong Yang ${ }^{2}$, \\ Hongwei $\mathrm{Mo}^{3}$, and Xianglin Huang ${ }^{1}$ \\ 1 School of Computer Science and Software, \\ Communication University of China, Beijing, China \\ \{lijianyu, lvrui, yangzx, huangxianglin \}@cuc.edu.cn \\ 2 School of Computer and Information Technology, Beijing \\ Jiaotong University, 100044 Beijing, China \\ yangshuzhong@163.com \\ 3 School of Automation, Harbin Engineering University, 150001 Harbin, China
}

\begin{abstract}
In this paper we present a new method to create small world networks based on K-means clustering analysis. Because of the close relationship between the small world networks and the data with clustering characteristics, the resulting networks based on K-means method have many properties of small world networks including small average distance, right skewed degree distribution, and the clustering effect. Moreover the constructing process also has shown some behaviors including networks formation and evolution of small world networks.
\end{abstract}

\section{Introduction}

In recent years, the discovery of small-world, scale-free and community properties of many natural and artificial complex networks has stimulated a great deal of interest in studying the underlying organizing principles of various complex networks, which has led to dramatic advances in this emerging and active field of research [1, 2, 3, 4, 5.

Considering the features of complex networks, a novel idea about how to construct the networks is to find the relationship between networks and data with clustering features. Unlike the model of Watts and Strogatz [1, we find an alternate route to generate the complex networks, especially to construct the small-world networks based on k-mean cluster analysis.

Many data sets from nature and society have the clustering characteristics. Like most data sets, most real networks also have the clustering property, especially in social networks [1]. In social networks, there are usually some groups of nodes (also called communities or modules), where nodes in each group are more likely connected with each other than to the rest of the networks [6]. One question could be given: "Could clustering analysis be used to construct relationships between them? Could networks be generated using data cluster analysis? From the above analysis, cluster analysis method (K-means method) could serve as a bridge exploring the relationship between them, and construct the networks based on the given data. 


\section{The New Method Based on KM Algorithm}

Procedure of our method based on k-means algorithm is given as below:

1. Place $k$ points into the space represented by the objects that are being clustered. These points represent initial group centroids;

2. Assign each point to the group that has the closest centroid;

3. When all points have been assigned, recalculate the positions of the $k$ centroids. Find the points nearest to the centroids and connect the nearest points obtained at the $i t h$ and the $(i+1) t h$ step in the same cluster. The measurement used here is Euclidean distance;

4. Repeat Steps 2 and 3 until the centroids no longer move. This produces a separation of all the points into groups from which the metric to be minimized can be calculated;

5. Repeat Steps 1, 2, 3 and 4 until all the possible and different initial $C_{n}^{k}$ cases are carried out.

Remark 1. There exist many factors, which affect the clustering analysis results such as number of clusters, the algorithm (k-means or fuzzy k-means method may be used), the data distribution, and the measurement of the data. Since the resulting complex networks are generated by the K-means method, the structure and topology of the networks will be affected by these factors.

\section{Properties of the Resulting Networks}

\section{The properties of the clustering path}

In order to analyze the networks in detail, all the clustering paths are to be divided into two classes. One is called local clustering path whose vertices belong to the same cluster, the other is called global clustering path whose vertices come from different clusters, see Fig.1. Generally the local paths are relatively short and won't fluctuate remarkably, at the end it terminates near the same cluster centroid. The global paths are long and they will go through the points which belong to other clusters, but later it will become stable and stop near one cluster centroid. From above analysis, we can conclude the paths show clustering properties. In any cases, because of the feature of K-means method, all paths avoid the long range connections and the global paths play the role of shortcuts to connect different clusters.

\section{Statistics properties of the networks}

In this part we will investigate properties of the resulting networks. We quantify the structural properties of these networks by their characteristic path length $L$, clustering coefficient $C$, and degree distribution $P(k)$. The characteristic path length, $L$, is the path length averaged over all pairs of nodes. The path length $d(i, j)$ is the number of edges in the shortest path between nodes $i$ and $j$. The clustering coefficient is a measure of the cliqueness of the local neighborhoods. For a node with $k$ neighbors, then at most $k(k-1) / 2$ edges can exist among 


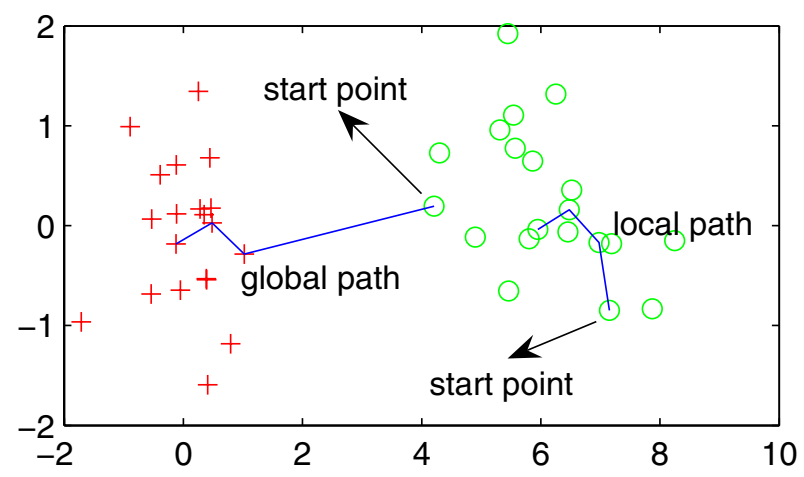

Fig. 1. The resulting clustering paths. Here the data consist of two classes of points which are represented by the plus $(+)$ and circle (o) sign.

them. The clustering of a node is the fraction of these allowable edges that occur. The clustering coefficient, $C$, is the average clustering over all the nodes in the graph. The degree of a vertex in a network is the number of edges incident on (i.e., connected to) that vertex. We define $P(k)$ to be the fraction of vertices in the network that have degree $k$.

\section{Example 1}

The data $(x, y)$ was generated from two bivariate independent Gaussian distributions $\left(X_{1}, X_{2}\right), X_{i} \sim N\left(\mu_{i}, \sigma_{i}\right)$ and $\left(Y_{1}, Y_{2}\right), Y_{i} \sim N\left(\mu_{i}, \sigma_{i}\right), i=1,2$ with different means $\mu_{1}=0, \mu_{2}=3$, and same variances $\sigma_{1}=\sigma_{2}=1$. Half of the data was generated from $\left(X_{1}, X_{2}\right),\left(Y_{1}, Y_{2}\right)$ respectively.Hence the number of clusters is two.

The average path length $L$ of the resulting networks is close to that of a random graph with the same size and average degree, 2.4 compared with 3.07, but its clustering coefficient $C$ is more than 20 times higher than a random graph, 0.4005 vs. 0.0175 . From the analysis, the resulting networks show small world feature.

In Fig.2(a), we show the networks' degree distributions. The degree distributions of the networks are often highly skewed and differs from the Poisson distribution. It's at the border between Poisson and power law distribution.

\section{Example 2}

The Data $\left(x_{1}, x_{2}\right)$ was generated from independent bivariate Gaussian distributions $\left(X_{1}, X_{2}\right), X_{i} \sim N(\mu, \sigma)$ with different means $\mu=5$ and variances $\sigma=1$, and independent bivariate standard Cauchy distributions $\left(Y_{1}, Y_{2}\right)$, the probability density function of is

$$
\frac{1}{\pi \gamma\left[1+\left(\frac{x-x_{0}}{\gamma}\right)^{2}\right]}
$$

where $x_{0}=0, \gamma=1$. The data has 400 points, and 200 points was obtained from $\left(X_{1}, X_{2}\right),\left(Y_{1}, Y_{2}\right)$ respectively. 


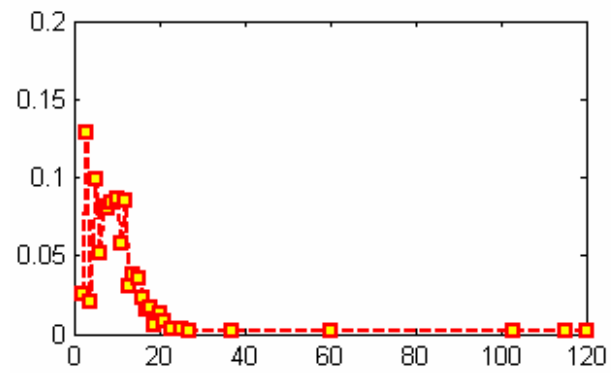

(a)

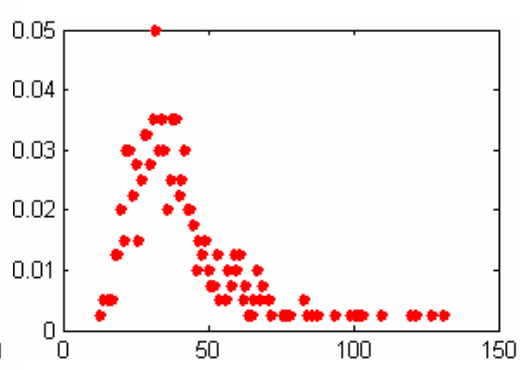

(b)

Fig. 2. (a)The degree distribution of the data with 600 points shown in example 1, (b)The degree distribution of the data with 600 points shown in example 2

This resulting graph has a small world structure, with: $C=0.5004>>$ $C_{\text {rand }}=0.04$, and $L=2.9270$ and $L_{\text {rand }}=3.3219$ are very close.

The degree distribution in Fig.2(b) showed Poisson distribution feature with a long tail.

\section{Conclusion and Research in the Future}

In this paper we presented the method based on k-means clustering analysis to create the networks. Although it is easy to understand the method, the idea is novel. We use the clustering thought which may be the nature of the universe and construct the relationships between data and networks. The experiments show that the generating networks has many properties which distinguishes itself from other existing complex networks. The generating networks can describe many phenomena of complex networks such as small world effect, and the evolution behavior. Moreover, a good and proper explanation for the formation of the small world networks topology is presented due to the given data's clustering feature. Finally, in future work, the factors that influence the formation and topology of the resulting networks will be discussed.

\section{References}

1. D. J. Watts et al: Collective dynamics of 'small-world' networks. Nature 393, 440-442 (1998).

2. S. N. Dorogovtsev et al: Evolution of networks. Advances in Physics 51, 1079-1187.

3. M. E. J. Newman: The structure and function of complex networks. SIAM Review 45, 167-256 (2003).

4. A.L. Barabási and R. Albert: Emergence of scaling in random networks. Science, 286, 509-512 (1999).

5. A. R. Barabási, A.L. and H. Jeong: Scale-free characteristics of random networks: The topology of the World Wide Web. Physica A 281, 69-77 (2000).

6. M. E. J. Newman and M. Girvan: Finding and evaluating community structure in networks. Phys. Rev. E 69:026113 (2004). 